\section{Tipologías y argumentos en torno a las técnicas de reproducción asistida en habitantes de Santiago de Chile}

\author{
FLORENCIA HERRERA $^{1, \mathrm{a}}$, BERTA TEITELBOIM ${ }^{1, \mathrm{~b}}$, \\ FERNANDO ZEGERS-HOCHSCHILD ${ }^{2}$
}

\section{Typologies and arguments about the use of assisted reproductive technologies among people living in Santiago, Chile}

Background: Assisted reproductive technologies still generate debate about ethical moral and religious issues. Aim: To analyze the opinions of the general public about the use of assisted reproduction technology (ART). Material and Methods: This analysis used the information gathered in a survey about human reproduction and modern reproduction technologies applied to a random sample of 1,500 volunteers aged 18 to 65 years, living in Santiago, Chile. Also, data from 25 structured interviews and six focal groups of eight participants each was used. To typify opinions concerning the use of assisted reproductive technologies (ART), a multiple correspondence analysis was undertaken. Results: People that support ART are non-religious young people of medium-high socioeconomic level. The arguments most commonly used by people to justify their positions about ART are the right to have children, the protection of kinship, that ART implies commercialization and manipulation of human life, the need to obey God's will, and the autonomy of informed and educated adults. Conclusions: The study allows us to understand in more complex ways the positions about ART. Chile does not have a legislation that regulates the use of ART. Generating information on the public opinion about these techniques we hope to contribute to the debate about the access and regulation of new reproductive technologies in Chile.

(Rev Med Chile 2015; 143: 1527-1532)

Key words: Fertilization; Health surveys; Infertility.

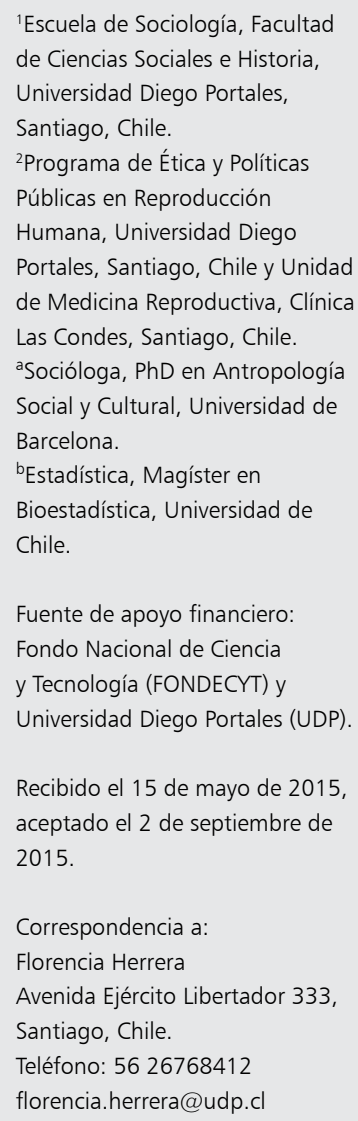

L as investigaciones que abordan las técnicas de reproducción asistida (TRA) desde la perspectiva de la opinión pública son escasas $^{1-3}$. En 2013 se publicó en la Revista Médica de Chile uno de los pocos estudios latinoamericanos que indaga sobre las posiciones respecto a las $\mathrm{TRA}^{4}$. En él se investigó la opinión de los habitantes de Santiago, Chile, respecto del uso de TRA a partir de los datos entregados por la Encuesta de Opinión Pública sobre Reproducción Humana y Usos de Tecnología Reproductiva Moderna (Encuesta) $)^{5}$.
El presente trabajo busca profundizar en las opiniones que despiertan las TRA en la población general a través de: 1) Elaborar tipologías de opinión; 2) Describir los argumentos que utilizan las personas para justificar estas opiniones.

\section{Material y Método}

Para efectos de esta publicación, dentro de las TRA, se incluyen exclusivamente las preguntas relacionadas con la fertilización in vitro (FIV) 
autóloga, es decir, con gametos de la propia pareja. En esta investigación, se utilizan metodologías cuantitativas y cualitativas.

El análisis estadístico consideró la construcción de tipologías de opinión frente a las TRA. Para construir estas tipologías se utilizó análisis de correspondencia múltiple $(\mathrm{ACM})^{6}$, el cual es una técnica de análisis factorial exploratoria para datos multivariados que se utiliza cuando las variables son categóricas. Este análisis busca describir, en un espacio de pocas dimensiones o factores, la estructura de asociaciones entre las categorías de las variables, así como las similitudes y diferencias entre los individuos a los cuales esas variables se aplican. Posteriormente se utilizó análisis de conglomerados para construir tipologías de opinión en base al $\mathrm{ACM}^{7}$. Para este análisis se utilizaron los datos entregados por la Encuesta ${ }^{5}$, cuyo trabajo de campo se realizó a finales de 2011. La Encuesta consideró una muestra aleatoria de 1.500 personas, representativas de la población entre 18 y 65 años que reside en el Gran Santiago. Más información sobre la metodología usada, puede encontrarse en el artículo publicado en la Revista Médica de Chile ${ }^{4}$.

Para comprender los argumentos que fundamentan las posiciones respecto a las TRA, se utilizó una metodología cualitativa. Se analizaron los datos provenientes de 25 entrevistas semiestructuradas ${ }^{8,9}$ y 6 grupos focales de 8 participantes cada uno ${ }^{10}$. Los entrevistados y participantes en los grupos se seleccionaron a través de un muestreo teórico intencionado, utilizando como criterios de selección las variables que, de acuerdo con la Encuesta, influyen en la opinión respecto a las TRA: edad, género, religión, grupo socioeconómico (GSE) y posición política. Las entrevistas se realizaron en octubre y noviembre de 2012 y los grupos en mayo y junio de 2013.

En las entrevistas y los grupos focales se utilizó una guía de preguntas que indagaba sobre las posiciones de los participantes respecto a la FIV. Esta pauta se aplicó de manera flexible, para dejar espacio a temas emergentes ${ }^{8}$. Las entrevistas y los grupos fueron grabados y luego transcritos textualmente.

Las transcripciones fueron analizadas cualitativamente. Se identificaron los segmentos más significativos de los datos y se crearon categorías temáticas a partir de ellos. Este proceso se conoce como 'codificación' ${ }^{11}$ y fue realizado con la ayuda del software de análisis cualitativo Nvivo. El análisis de las transcripciones permitió identificar los argumentos en torno a las TRA más prevalentes en entrevistas y grupos.

El estudio fue aprobado por el Comité de Ética de Investigación de la Universidad Diego Portales. Los participantes de encuesta, entrevistas y grupos firmaron un consentimiento informado donde se establecía que su participación sería voluntaria y anónima. Se utilizaron seudónimos para resguardar la identidad.

\section{Resultados}

\section{Tipologías de opinión respecto a las TRA}

Se consideraron para el análisis de correspondencia múltiple las variables sociodemográficas: sexo, edad, hijos, GSE, religión y posición política, $\mathrm{y}$ un grupo de preguntas del cuestionario acerca de la aprobación o no del uso del FIV.

Se construyeron tipologías de opinión frente al apoyo o no de la FIV, en base a las distancias de las opiniones representadas en la Figura 1, encontrándose 6 grupos marcadamente definidos:

Grupo 1: Totalmente de acuerdo con FIV. Representó a $24,1 \%$ de los encuestados, se caracterizaban por ser personas jóvenes, no religiosas y que pertenecen a GSE medio-alto.

Grupo 2: Apoyo a FIV con restricciones en el acceso. Representó a 35,2\% de los encuestados. Apoyaban el uso de FIV sólo para mujeres en edad fértil y parejas heterosexuales. Las personas en este grupo tendían a pertenecer al GSE medio-alto y se concentraban entre los 45 y 65 años. Eran mayoritariamente mujeres.

Grupo 3: Sin opinión clara. Representó a 18,9\% de los encuestados. Se caracterizaban por tener bajo nivel educacional y ser mayores de 45 años. Tendía a haber una mayor presencia de católicos no religiosos.

Grupo 4: Católicos reticentes al uso de FIV. Representó a 8,5\% de los encuestados. Eran reticentes al uso de FIV, pero aceptaban su uso por parte de parejas heterosexuales casadas. Tendían a tener educación media completa. 


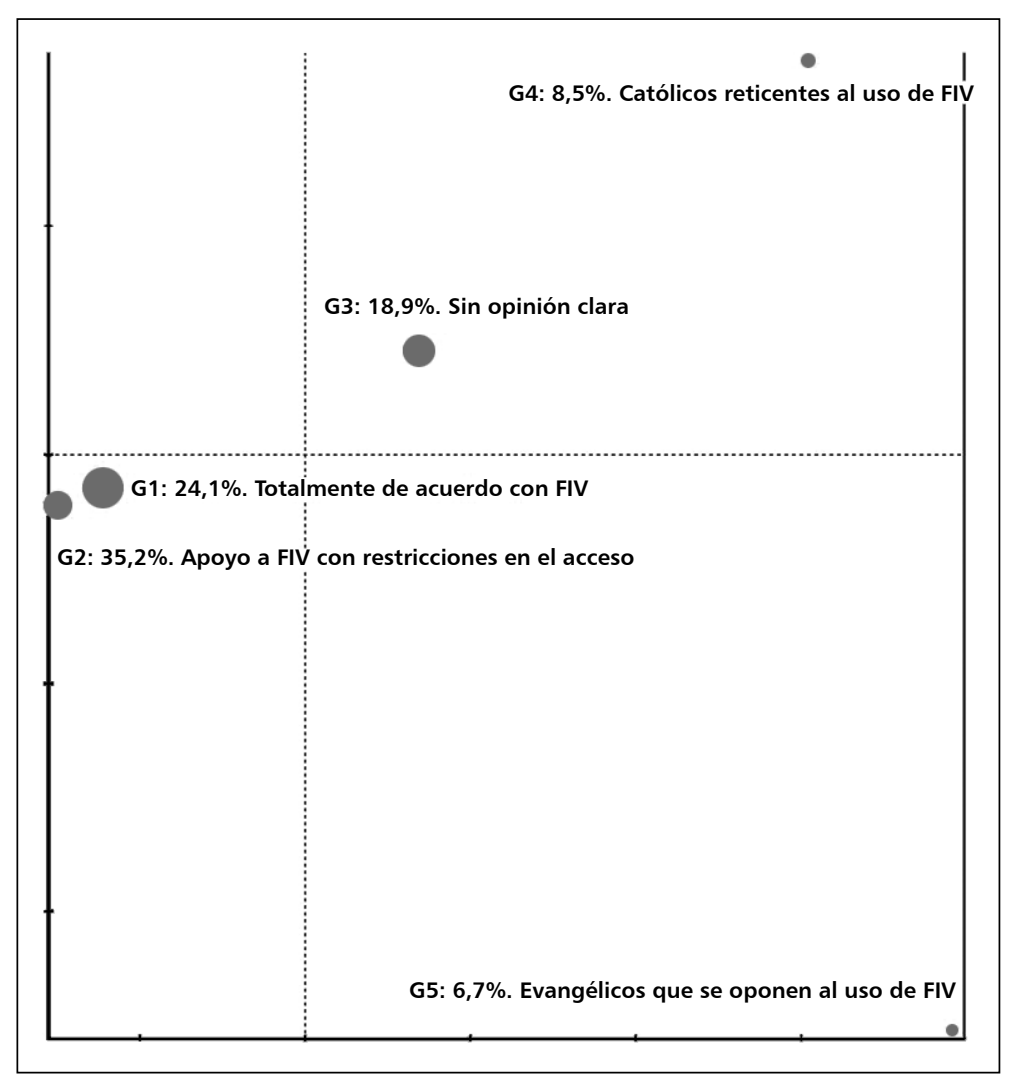

Figura 1. Tipologías de opinión. Para construir las tipologías se utilizaron 44 variables y 81 categorías de la Encuesta de Opinión Pública sobre Reproducción Humana y Usos de Tecnología Reproductiva Moderna ${ }^{5}$.
Grupo 5: Evangélicos que se oponen al uso de FIV. Representó a 6,7\% de los encuestados. Las personas en este grupo se opusieron a la FIV en todos los casos. Tendían a ser mayores de 45 años y posición política de derecha.

Grupo 6: No responden a las preguntas sobre el uso de FIV. Representó a 6,5\% de los encuestados. Tenían un bajo nivel educacional y en general eran mayores de 45.

\section{Argumentos en torno a las TRA}

$\mathrm{Al}$ analizar los contenidos de las entrevistas y los grupos focales se pudo observar que las posiciones respecto a las TRA eran más complejas que lo que refleja la Encuesta. En entrevistas y grupos, los participantes pueden exponer sus opiniones y justificarlas, no sólo manifestar su apoyo o rechazo a la FIV. A continuación se presentan los argumentos más utilizados por las personas para justificar sus posiciones respecto a las TRA.

\section{El derecho a tener hijos}

Este es el argumento más prevalente. La mayoría de los participantes considera que dar vida es un valor trascendental y los hijos son siempre algo positivo. Se apoya el uso de FIV porque se considera que tener hijos es un derecho de toda persona y que nadie debería tener impedimentos si desea convertirse en madre o padre. Incluso, algunos participantes argumentan que al ser niños muy buscados y deseados, los hijos producto de TRA serán especialmente queridos y cuidados. Un participante usa las siguientes palabras para manifestarse a favor de las TRA: "Yo creo que es un derecho humano y el objetivo es algo tan bello como tener un hijo" (Grupo Focal 4, 30-44 años, GSE bajo, sin religión). Al preguntar si apoya el uso de FIV una entrevistada responde:

"Si hay dos personas que van a poder darle una vida rica y esperan a un niño, ¿por qué no?, por eso, por amor, por querer traer un niño al mundo, eso es súper importante, dar vida es súper importante $y$ 
súper lindo, ¿por qué vas a negar eso?" (Entrevista a Catalina, 26 años, GSE medio, sin religión).

\section{Protección familia conyugal}

Muy relacionada con el argumento recién expuesto, está la posición de personas religiosas (católicas y evangélicas) y mayores (45-65 años) que consideran que las TRA ayudan al fortalecimiento de la familia conyugal. Están de acuerdo con el uso de las TRA siempre y cuando sirva para que una pareja heterosexual casada e infértil pueda 'tener familia'. Las TRA no pueden ser la primera opción para tener hijos y tampoco deben poder acceder a ellas personas solas o parejas homosexuales. Un participante afirma: "Estoy a favor porque se conserva el núcleo familiar" (Grupo Focal 6, 45-65 años, GSE bajo, evangélico).

Las personas en esta posición tendían a temer que la masificación de las TRA tenga como consecuencia el debilitamiento de la familia como ellos la entienden -pareja heterosexual con hijos biológicos- al permitir que mujeres solas o parejas homosexuales tengan hijos. Estas personas suelen estar en contra de la donación de gametos y embriones.

\section{Comercialización y manipulación}

Las personas con una posición más reticente frente a las TRA argumentan que el desarrollo de la medicina reproductiva abre las puertas a que se comercialice y se manipule la vida humana. Un participante sostuvo: "Ahí se pierde el amor y se convierte en materialismo. Es una compra” (Grupo Focal 6, 45-65 años, GSE bajo, evangélico). Este argumento está más presente entre participantes religiosos, pero también lo utilizan personas con otros perfiles. Otro participante sostuvo: "Si se crea la tecnología, se podría crear como para hacer una industria que vende guaguas" (Grupo Focal 1, 1829 años, GSE alto, sin religión). Un entrevistado sostuvo que 'no le gusta' la FIV porque es "llevar al humano a algo parecido a un producto" (Entrevista a Juan Ignacio, 28 años, GSE medio).

\section{La voluntad de Dios}

Los entrevistados que se manifestaron en contra de las TRA consideraban que las personas que no han podido tener hijos de forma natural deben resignarse. Utilizar la medicina reproductiva para concebir implicaría 'ir en contra de la voluntad de
Dios'. La utilización de este argumento fue muy común entre las personas evangélicas. Un participante sostuvo: "Uno tiene que aceptar lo que Dios quiere en el sentido natural. Si yo no puedo tener hijos, tendría que aceptar no más" (Grupo Focal 6, 45-65 años, GSE bajo, evangélico).

\section{Autonomía para decidir}

Muchos participantes señalaron que, independiente de su posición personal respecto a las TRA, cada persona debiera tener la libertad para elegir qué camino tomar al momento de enfrentar dificultades para concebir. Carla afirmó: "En cuanto a si otras personas lo hagan o no lo hagan, yo de verdad creo que cada uno elige" (Entrevista, 20 años, GSE medio, sin religión).

\section{TRA como algo antinatural}

Algunos participantes sostuvieron que los hijos deben nacer del amor, para ellos esto implica que sean concebidos mediante el acto sexual. Las TRA son 'antinaturales', ya que intervienen en el proceso natural del sexo y el amor y lo transforman en algo frío y artificial. Consuelo vincula el amor con lo natural:

"Claramente yo quiero vivir el proceso lo más natural posible, que ojala sea con mi pareja, con el amor de mi vida y que nuestros hijos sean el fruto de nuestro amor y que los criemos juntos" (Entrevista, 23 años, GSE medio, sin religión).

\section{Adopción versus reproducción asistida}

Muchos participantes mencionaron espontáneamente la alternativa de la adopción cuando se les preguntó qué debería hacer una pareja infértil. Estas personas no se manifiestan necesariamente en contra de las TRA, pero dejaron claro que piensan que el camino más adecuado es la adopción. Varios participantes mencionaron que ellos no utilizarían TRA para tener hijos, porque hay muchos niños abandonados que necesitan una familia. Posicionan a la adopción como la alternativa 'altruista' y, en contraposición, a las TRA como la 'egoísta'. Pedro explicó que para él la FIV sería una segunda opción: "No la descarto, solamente la tomaría como segunda opción, en el sentido de que prefiero darle la ayuda a un niño que de verdad lo necesite y que no tenga papá y que lo esté buscando" (Entrevista, 20 años, GSE medio, sin religión). 


\section{Discusión}

Los resultados de este estudio son consistentes con los de otras investigaciones similares. En un estudio sobre actitudes respecto a las TRA en Estados Unidos de Norteamérica, Shreffler et $\mathrm{al}^{2}$ encontraron que las inquietudes éticas respecto a las TRA crecían a medida que aumentaba la edad y disminuía el nivel socioeconómico. Dempsey y Critchley ${ }^{12}$ proponen una tipología similar a la propuesta aquí, basándose en los resultados de una encuesta realizada en Australia. El grupo que mostraba mayor comodidad con las TRA estaba compuesto mayoritariamente por mujeres, con educación universitaria (relacionado con GSE en Chile), jóvenes y poco religiosas. En los grupos más reticentes tendía a haber mayor presencia de personas mayores y religiosas.

Los argumentos identificados en entrevistas y grupos también han sido revelados por investigadores en otros países. Luna ${ }^{13}$ sostiene que un argumento a favor de las TRA es el de la 'ayuda a la maternidad y paternidad', donde se considera que tener hijos biológicos es una necesidad y un derecho de los seres humanos. Y un argumento que justifica el rechazo a las TRA es el "argumento de lo natural" donde se enfatiza lo artificial de las TRA esgrimiendo que la gestación se realiza por medios tecnológicos y, por lo tanto, sin amor. Luna también incluye el "argumento de la posibilidad de adopción” entre los argumentos utilizados para rechazar el uso de las TRA.

Evans $^{14}$, al estudiar las opiniones de personas religiosas en Estados Unidos de Norteamérica, identifica el discurso de la 'dignidad y equidad humana' para oponerse a las TRA -que considera que las TRA implican la manipulación de embriones, la selección de material genético y la comercialización de la reproducción- y el discurso "fatalista prometeico" que argumenta que Dios tiene un plan para cada ser humano y este plan no debería ser intervenido.

El análisis de las tipologías de opinión y de los argumentos en torno a las TRA ayuda a develar las ambivalencias que existen detrás del apoyo mayoritario a la FIV (71,8\% de acuerdo a la Encuesta) en Chile 5 . Aproximadamente un cuarto de los encuestados están a favor del uso de FIV sin ninguna restricción. Los argumentos que respaldan esta posición son que tener hijos es un derecho de toda persona y que todos deben tener la autonomía para decidir.
Otro grupo importante se manifiesta a favor de las TRA, pero piensa que sólo se deben usar para fortalecer la familia conyugal heterosexual. Más que estar en contra de las TRA, estas personas están en contra del surgimiento de otras formas familiares (homoparentales, monoparentales).

El rechazo a las TRA es minoritario, y puede explicarse en gran parte por razones religiosas ('no se debe ir en contra de la voluntad de Dios') y por la asociación entre concepción, naturaleza y amor ('TRA como algo antinatural').

En las entrevistas y grupos de discusión se puede identificar una posición que no es tan clara en los resultados de la Encuesta y las tipologías de opinión. Se trata de personas reticentes al uso de las TRA porque estiman que estas implican la comercialización y manipulación de la vida humana. Este argumento manifiesta el temor que existe a lo que puede pasar si las TRA no son reguladas, más que un rechazo a las TRA en sí mismas.

Mediante la combinación de herramientas cuantitativas y cualitativas, este estudio permite comprender las posiciones en torno a las TRA. A diferencia de otros países, como Argentina y Uruguay, Chile no cuenta con una legislación que regule las TRA. A través de la entrega de información sobre qué piensan los chilenos en relación a estas técnicas, se espera contribuir al debate sobre el acceso y regulación de las nuevas tecnologías reproductivas en Chile.

Agradecimientos: Los hallazgos que se presentan fueron obtenidos de una investigación realizada en Santiago de Chile, financiada por el Fondo Nacional para el Desarrollo de la Ciencia y la Tecnología (Proyecto FONDECYT No 11110287). Se agradece el apoyo del Fondo Nacional para la Ciencia y la Tecnología y del Programa de Ética y Políticas Públicas en Reproducción Humana (PREPRE) de la Universidad Diego Portales. Asimismo, se agradece la valiosa ayuda de Yanko Pavicevic, ayudante de investigación, en la sistematización de los datos.

\section{Referencias}

1. Johnson KM, Simon RM. Women's Attitudes Toward Biomedical Technology for Infertility: The Case for Technological Salience. Gend Soc. 2012; 26 (2): 261-89. 
2. Shreffler KM, Johnson DR, Scheuble LK. Ethical problems with infertility treatments: Attitudes and explanations. Soc Sci J. 2010; 47 (4): 731-46.

3. Hudson N, Culley L, Rapport F, Johnson MR, Bharadwaj A. "Public" perceptions of gamete donation: a research review. Public Underst Sci. 2009; 18 (1): 61-77.

4. Herrera F, Teitelbom B, Russo M, Salas SP, Zegers Hochschild F. Encuesta de opinión pública sobre reproducción humana y usos de tecnología reproductiva asistida en habitantes de Santiago, Chile. Rev Med Chile 2013; 141 (7): 853-60.

5. Herrera F, Teitelboim B, Salas S, Zegers Hochschild F. Encuesta de Opinión Pública sobre Reproducción Humana y Usos de Tecnología Reproductiva Moderna. Santiago, Chile: Ediciones Universidad Diego Portales; 2013.

6. Peña D. Análisis de Datos Multivariantes. Mc Graw Hill/ Interamericana de España. 2002. 529 p.

7. Moscolini, N. Las Nubes de Datos. Métodos para Analizar la Complejidad. Rosario: Universidad Nacional de Rosario; 2011.
8. Mason J. Qualitative Researching. SAGE Publications; 2002.

9. Flick U. Introducción a la investigación cualitativa. Madrid: Morata; 2004.

10. Canales M. Metodología de la Investigación Social. Introducción a los oficios. Santiago de Chile: Ediciones LOM; 2006.

11. Atkinson P, Coffey A. Encontrar sentido a los datos cualitativos: estrategias complementarias de investigación. Alicante: Universidad de Alicante; 2005.

12. Dempsey D, Critchley C. Comfort with use of Assisted Reproductive Technologies (ART) for family formation by same-sex and heterosexual couples: A survey of Australian social attitudes. Gay Lesbian Issues Psychol Rev. 2010; 6 (2): 90-102.

13. Luna F. Reproducción asistida, género y derechos humanos en América Latina. San José, Costa Rica: Instituto Interamericano de Derechos Humanos; 2008.

14. Evans JH. Contested Reproduction: Genetic Technologies, Religion, and Public Debate. Chicago: University of Chicago Press; 2010. 